\title{
Comparison of MANET self-organization methods for boundary detection/tracking of heavy gas cloud
}

\author{
Mateusz Krzysztoń \\ Institute of Control and Computation Engineering, Warsaw University of Technology \\ Nowowiejska 15/19, 00-665 Warszawa, Poland \\ Email: mateusz.krzyszton@gmail.com
}

\begin{abstract}
Mobile wireless ad hoc network (MANET) becomes increasingly popular in responding to emergency situation. In this paper a possibility to support rescue team in monitoring heavy gas cloud with MANET comprised of mobile sensing devices is investigated. In the view of the current state of research, two methods for controlling mobile sensing devices during MANET self-organization are presented. The first one is based on a greedy approach whereas the second on a repulsion from the estimated centroid of a cloud and other nodes. Various variants of both methods are considered and their efficiency in terms of detection quality and energy saving is evaluated with MobASim simulation software. The results are discussed and one variant is chosen as the basis for the future research.
\end{abstract}

\section{INTRODUCTION}

A LL over the world great amount of toxic substances is transported and stored. Some of these substances after release form clouds of gas heavier than air [1]. Despite high safety standards severe accidents, in which dangerous substance is released to the atmosphere, occur. Examples of accidents involving heavy gas release include those with chlorine [2], nitrogen dioxide [3] and sulfur dioxide [4]. Heavy gas cloud can be created by natural reasons as well - in 1986 a massive, sudden release of carbon dioxide occured from Lake Nyos, a volcanic crater lake, and as a result around 1700 people were killed [5]. Another source of the toxic gas cloud can be military or terrorist attack [6], [7].

As heavy gas cloud is formed a rescue team has two goals: evacuate endangered area and neutralize the cloud. In both knowledge of position, boundary and direction of the cloud is substantial as it supports rescue team in surrounding and then suppressing the cloud, making decision which area to evacuate first and identifying safe evacuation routes. Usually there is no need for modeling exact concentration level of gas inside the cloud.

Because of the negative buoyancy behavior of the heavy gas cloud is different than the one showed by positively or neutrally buoyant clouds. The main difference is gravitational velocity field (gravitational slumping) which influences the way cloud moves and changes its shape with time. Hence, special group of mathematical models was developed to describe the dispersion of heavy gas in the atmospheric air [8]. However, usage of these models to define boundary and position of the cloud demands specifying values of many parameters as direction and speed of wind, quantity of released gas, type of release (instantaneous or continuous), etc., which are often unknown and/or variable. Most of the described models assume obstacle-free, flat terrain; Even if obstacles or topography of terrain is considered only simple scenarios can be modeled. These issues create need for more universal, environment independent methods for the cloud boundary tracking in an unknown terrain.

In this work implementation of Mobile Ad Hoc Networks (MANET) for supporting rescue team in emergency action is proposed. MANET is comprised of wireless mobile devices, which can dynamically and autonomously self-organize into temporal networks to provide a discovering and tracking boundaries of dynamic heavy gas cloud. The network adapts to current conditions in deployment area by forming adequate topology. Nodes communicate wirelessly to exchange their knowledge about the environment.

The article is organized as follows. First, state of the current research on applying MANET in emergency situations and methods for boundary detection/tracking is presented. Then in the section III problem is formulated. Next two simple distributed methods for boundary of heavy gas cloud discovering and tracking are proposed. In the section $\mathrm{V}$ different variants of these methods are evaluated and compared in terms of both quality and energy efficiency. Finally, results are discussed and future directions of work are briefly outlined.

\section{RELATED WORK}

Contemporary lots of research focus on both using sensor networks to support detection and response to emergency situations and on detecting boundary of phenomena. Below the most interesting works in these two fields are briefly presented.

MANET can be used in emergency situation to address various issues. Usually as the result of natural disaster communication systems are down [9]. Thus MANET can be used to establish new communication layer for rescue team [9], [10]. In [11] system for firefighters that provides possibility to conduct audio and video conference during action is described. Another communication network architecture is presented and assessed for existing Telemedicine Service in [12]. In [13] MANET is created to establish communication with single robot that searches building in emergency scenario. The comparison of the MANET based solution with the static network in terms of throughput shows improvement 
if network's topology does not change often, but decrease otherwise. The influence of relay nodes placement on network reliability, stability and availability is discussed in [14].

Another purpose of MANET can be supporting decision making process of emergency team by providing important data. In [15], network is created to serve as a communication layer and collect medical data from remote sources about mass casualty incidents, thus supports decision making. Similar scenario of MANET application is presented in [16] - locations and current state of victims are monitored and provided to rescue team. Another application of MANET is to support evacuation of people from endangered area by providing an appropriate evacuation route in real time [17], [18].

MANET can be also used in disaster detecting — methods based on analyzing people behavior are proposed in [17], [19], whereas distributed control system for maximizing the joint detection probability of events in a given area is presented in [20].

In the same time much effort has been spent on research on detecting and tracking phenomena clouds - events characterized by nondeterministic, dynamic temporal variations of cloud shape, size, speed, and direction of motion along multiple axes [21]. The examples of phenomena cloud are oil spills, movement of group of people and gas clouds. Most interesting approaches are presented below, divided according to the type of used devices - stationary and mobile (can move autonomously).

A number of works on phenomena cloud detection with stationary nodes exists. In [22] authors focus on meeting time and accuracy constraints in a task of tracking phenomena. In [23], scenario with nodes sparsely deployed in the area is considered. In such situation collected data can be ambiguous in terms of number and shape of detected phenomena clouds. To reconstruct contours method based on gradients of concentration is designed.

Important issue in WSN networks is energy saving. In [24], method for limiting power usage by selecting subset of WSN nodes that are localized on the boundary of phenomena (representative nodes) and reducing size of messages is proposed. Distributed boundary estimation strategy with mechanisms for decreasing number of messages sent between nodes, and adaptive turning off sensors, is described in [25]. In [21] authors propose several distributed methods and focus on minimizing resource utilization by both reducing number of active sensors and optimization of centralized query processor, which gather information about cloud in real time. Energy efficient algorithm for phenomena tracking for the case of void area (area not covered by nodes) existence is presented in [26]. Interesting scenario is considered in [27] — sensors are carried by people, thus nodes have no influence on their location, but are mobile. Authors address their method for scenarios with dense network, where lowering traffic is crucial aspect in terms of energy efficiency.

As concluded in work on target tracking [28] use of mobile devices can increase tracking performance significantly in comparison with stationary network of the same number of devices. Hence, much research on applying mobile devices has been conducted. Comprehensive survey on boundary estimation, covering and tracking with collaborative sensors can be found in [29]. In the next few paragraphs the most important approaches (including the newest research, not included in the survey) are discussed. In some works instead of detecting boundary more general task is considered - detection of perimeter defined as curve of the given constant concentration. Boundary can be seen as perimeter given by concentration close to zero.

Tracking and exploring phenomena cloud with one device is important issue in the first phase of detection, when phenomena cloud is sensed by one node only and other nodes did not arrived yet to the area of interest. The decision about the direction of movement can be based on trigonometric reasoning [30] or gradient of concentration [31]. Additionally, in [31] use of artificial neural networks for the nonholonomic mobile robot to move in the designed direction is described.

In [32] multiple autonomous vehicles are used to estimate boundary, however, with no cooperation between them. Authors focus on tracking algorithm, that enables each vehicle to go along boundary based on it's concentration measurements. Measurement noise is addressed. Data gathered from all vehicles is used to estimate boundary. The tracking algorithm was validated in static environment using testbed in [33]. The same algorithm was used in [34] to estimate phenomena cloud boundary with single mobile node. Additional support by WSN was introduced to correct mobile node's movement by predicting future center of the cloud.

Cooperation between robots can increase quality of cloud boundary detection. In [35], algorithm for even robots placement on the boundary was proposed. The algorithm was proved to converge in case of static boundaries and to be efficient for slowly-moving boundaries. However, assumptions that initial estimation of boundary is known to agents and each agent is able to locally estimate the tangent and the curvature of the boundary are needed. These assumptions are not valid in the case of heavy gas cloud, because robot can measure gas existence in one point only at the same time.

Another cooperation based method for placing evenly on the boundary was introduced in [36]. Additionally, group of mobile robots track perimeter of certain substance counterclockwise using camera. However, to uniformly distribute around perimeter the desired separation distance need to be set a priori.

An interesting method for the stationary environment, inspired by the active contour model used in the image segmentation field, was proposed in [37]. The approach is strictly dependent on the concentration of substance - it is assumed that lowest concentration is on the boundary and a gradient of concentration points to that boundary. Algorithm for scattering devices evenly, based on pairwise repulsion force, was additionally proposed. However, node needs to choose between tracking boundary or scattering in every step. Similar idea is presented in [38], where non-stationary environment is considered. Again, assumption on concentration is needed. 
Another method based on concentration value is described in [39]. Every sensor knows concentration in its location and in its close neighbourhood. To reach the location with the given concentration (concentration on the boundary) the sensor decides in every step which neighbouring location move to. Various strategies of taking decision were proposed and examined in scenarios with linear and non-linear variations of concentration.

In [40], group of unmanned underwater vehicles is used to find and patrol underwater perimeter. Two methods for controlling movement of vehicles were proposed and compared. The first one is based on aforementioned snake algorithm (concentration based). The second models vehicles as a gas of particles, which affects each other speed, similarly to the one of the approaches presented in this paper. The authors conclude that free-concentration methods are better for tracking underwater perimeter in real world scenarios.

Some research was conducted on using UAVs (unmanned aerial vehicles) to detect and track the shape of an environmental boundary. In [41], authors propose the method for calculating path of UAV that allows recognition of the contaminant cloud's shape. The approach was extended with methods for predicting contaminant cloud's shape [42] and avoiding obstacles [43]. UAVs can also be used to track forest fire boundary [44].

Interesting issue of the boundary detection is discussed in [45]. Authors propose some algorithms for deciding when to start spreading sensors (how far in advance before reaching the boundary) to speed up converge. The algorithm is based on a rate of concentration change, thus is not proper for situation in which boundary is defined with small concentration value (whole area where any gas exists should be classified as cloud interior). However, the idea of early sensor spreading should be addressed in our work in the future. Other drawbacks of proposed spreading algorithm is its centralized nature and assumption on obstacles free environment.

In the view of this brief review, MANET can be successfully used in emergency situation. At the same time there is lack of proper mobility control method for nodes for case of detecting boundary of dense gas cloud. Most of above methods base on concentration distribution, which is inadequate for scenarios with dense gas dispersion, because of various reasons. Firstly, heavy gas cloud has very dynamic internal changes. Secondly, gas sensors sense gas in single point only at a time and the sensory readings can be inaccurate. Additionally, even slightly pleated area influences distribution of gas in the area lower concentration can be caused by existence of both cloud boundary and hill. Hence, we propose two new methods for real time estimation of area covered by heavy gas cloud with MANET, based on binary information from sensors about gas existence.

\section{PROBLEM DESCRIPTION}

The aim of the paper is to create a sensing network for a heavy gas cloud detection in a two-dimensional workspace $W$, and boundary tracking to estimate a size of this. In our investigation, a network is a physical system modeled as a set of $n$ unmanned vehicles or mobile robots $D_{i}, i=1, \ldots, n$. All these vehicles are equipped with radio transceivers and gas sensors. All network nodes are solid bodies with an arbitrary shape. In order to simplify the description of the system we model each network node by a polygon with its reference point $\mathbf{c}^{i}=\left[x^{i}, y^{i}\right]$, which is the location of the device (exactly its antena). All vehicles are forced to move in advisable direction with the speed $v \in\left[v_{\min }, v_{\max }\right]$.

In this work it is assumed that each pair of nodes $D_{i}$ and $D_{j}$ can communicate independently on the Euclidean distance between these nodes $\left(d_{i j}\right)$, with use of external communication system. In the future, local multi-hop communication between each pair of nodes should be considered to enable deploying the network in area with no external connectivity system.

The goal of both methods is to control direction and speed of each node in such way, that would increase quality of estimation of area covered by gas and decrease total distance traveled by all nodes. The proposed methods are based on PFM (Potential Function Mobility) model described in details in [46]. In this model each node has single goal $g$ defined with target point in the workspace $\mathbf{c}_{g}^{i}=\left[x_{g}^{i}, y_{g}^{i}\right]$ and its movement is influenced by positions of other nodes and obstacles. The PFM model combines concepts of an artificial potential fields and particle-based mobility schemes. Each node is treated as a self-driven particle moving from a high-value state to lowvalue state of artificial potential field. The artificial potential function is constructed as a sum of repulsive and attractive potentials. Its value depends on Euclidean distance between a given node and all other nodes in the network, and the distance to target position and obstacles in $W$. As in this work obstacles-free environment is assumed each device $D_{i}$ has to calculate its new position solving problem of minimizing an artificial potential function $U^{i}$ (influence of obstacles is omitted):

$$
\begin{aligned}
& \min _{\mathbf{c}^{i}}\left[U^{i}\left(\mathbf{c}^{i}\right)=U_{g}^{i}\left(\mathbf{c}^{i}\right)+\sum_{j=1, j \neq i}^{n} U_{j}^{i}\left(\mathbf{c}^{i}\right)\right. \\
= & \left.\epsilon_{g}^{i}\left(\frac{\bar{d}_{g}^{i}}{d_{g}^{i}}-1\right)^{2}+\sum_{j=1, j \neq i}^{n} \epsilon_{j}^{i}\left(\frac{\bar{d}_{j}^{i}}{d_{j}^{i}}-1\right)^{2}\right],
\end{aligned}
$$

where $U_{g}^{i}$ and $U_{j}^{i}$ are potentials derived from $g$ and $D_{j}$, respectively. $\epsilon_{g}^{i} \geq 0$ and $\epsilon_{j}^{i} \geq 0$ are weighting factors determining the importance of, respectively, the goal $g$ and the device $D_{j} . d_{g}^{i}$ and $d_{i}^{i}$ are real Euclidean distances between $\mathbf{c}^{i}$ and respectively, $\mathbf{c}_{g}^{i}$ and $\mathbf{c}_{j}^{i}$ after a network transformation, and $\bar{d}_{g}^{i}$ and $\bar{d}_{j}^{i}$ the reference distances between $\mathbf{c}^{i}$ and respectively, $\mathbf{c}_{g}^{i}$ and $\mathbf{c}_{j}^{i}$. The reference distance is understood as the expected distance between node and target or other node, accordingly.

The final network topology depends on choice of target location $\left(\mathbf{c}_{g}^{i}\right)$, the values of the reference distances $\left(\bar{d}_{g}^{i}, \bar{d}_{j}^{i}\right)$ and weighting factors $\left(\epsilon_{g}^{i}, \epsilon_{j}^{i}\right)$. Hence, in the next section two distinct methods for calculating these values in task of estimating boundary of area covered by gas are presented. 


\section{Methods}

The detection of area covered by gas is divided into two phases. In the first one, when only one node detects heavy gas with sensor, the node has to track the cloud and broadcast information about its position to others. All nodes that do not sense gas are attracted by the point defined by the position of the node that senses gas. Tracking phase last to the moment in which at least one more node arrives to the area covered by gas. Then, the boundary detection phase starts.

The algorithm for controlling node that senses gas in the tracking phase is presented in Algorithm 1. The algorithm is executed as only the node starts to sense gas. The idea of the algorithm is to move inside the cloud as long as gas is sensed ( $k$ steps), then when the cloud is lost return to the cloud by taking opposite direction $(k / 2$ steps $)$ and choose new direction randomly. In this phase $\epsilon_{j}^{i}=0$ (thus $U^{i}=U_{g}^{i}$ ). Moving in the given direction is achieved by adding attracting goal $g$ in an appropriate location $\mathbf{c}_{g}^{i}$ with $d_{g}^{i}=1$. The advantage of this algorithm is simplicity and power efficiency thanks to little computation needed. More advanced approach, that does not base on gas concentration as well, was presented in [30].

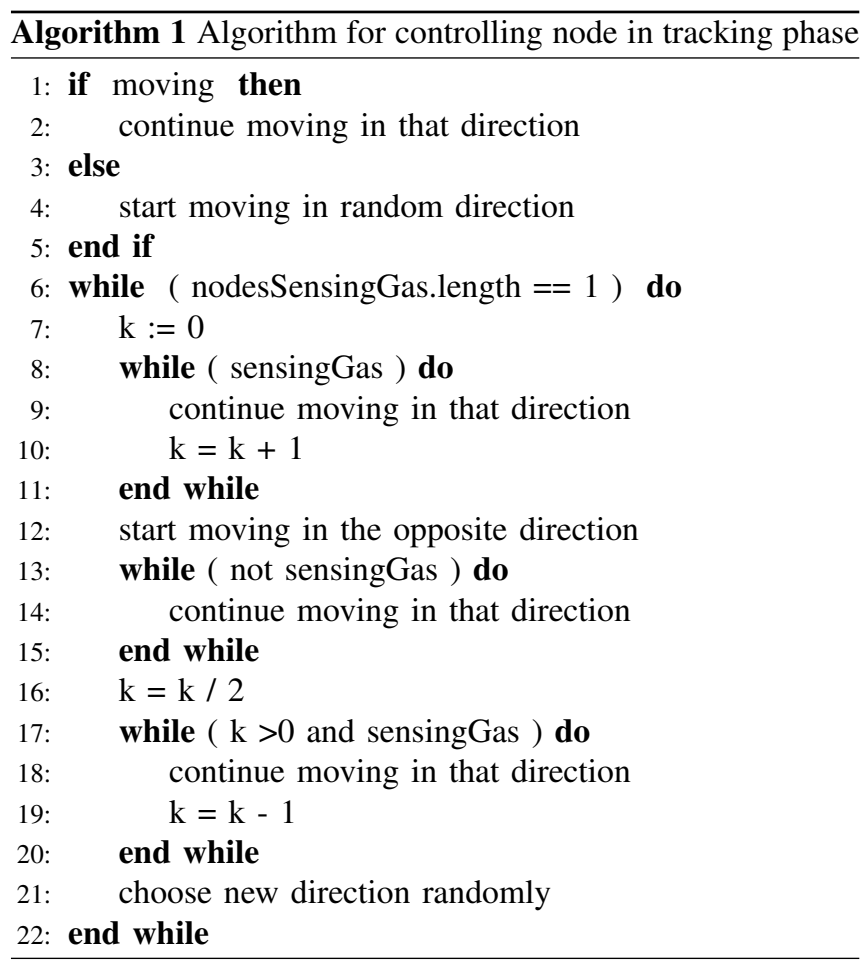

As at least two nodes sense gas the estimation area covered by gas phase begins. Below two different approaches for implementation of this phase are proposed. Before the approaches are described in details few remarks have to be made:

Remark 1. Binary sensory reading causes that all sensors that do not sense gas in a given moment are treated the same way, independently on how many steps have passed after last positive sensory reading. Such simplification causes that much of important information can be lost from the perspective

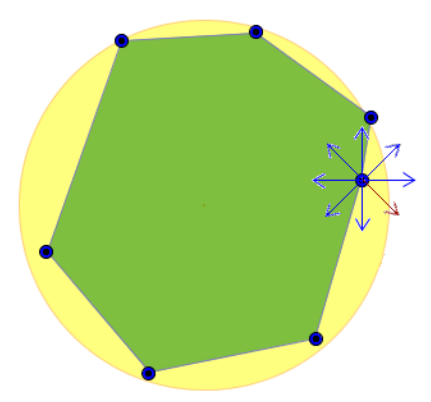

Fig. 1: Directions of movement considered by a node in the greedy approach $\left(k_{d}=8\right)$.

of rescue team. Hence, three different states of node are introduced:

(a) sensing gas at time $t$

(b) not sensing gas at time $t$, but did sense gas at least once in last $h$ steps

(c) not sensing gas at time $t$, and did not sense gas in any of last $h$ steps

The boundary of the covered area is estimated as convex hull of the set $C^{+}$defined as the set of locations of all nodes in state (a) or (b) with $h=2$.

Remark 2. The term centroid will refer to the center of the cloud $\mathbf{c}_{c}$, which is calculated as an arithmetic average of positions of all nodes that sense gas:

$$
\mathbf{c}_{c}=\frac{\sum_{\mathbf{c}^{i} \in C^{+}} \mathbf{c}^{i}}{\left|C^{+}\right|} .
$$

Remark 3. In both approaches the nodes that do not sense gas are attracted by single goal located in centroid $\left(\mathbf{c}_{g}^{i}=\mathbf{c}_{c}\right)$ with reference distance $d_{g}^{i}=1$ and influence of other nodes is omitted $\left(\epsilon_{j}^{i}=0\right)$.

\section{A. Greedy approach}

In greedy approach every node in every time step chooses one of $k_{d}$ directions to move. The decision is based on localization of all other nodes that sense gas $\left(C^{+}\right)$. The node calculates the area of current cloud's shape estimation (convex hull of $C^{+}$), simulates the move in each of $k_{d}$ directions and calculates the change of area after each move. In accordance to greedy approach the direction that increases the area most is chosen as a new direction of the node's movement. However, as decision is independent on the simultaneous decisions of other nodes, it may occur that the chosen direction is not optimal in the given situation. As in the exploration phase moving in the chosen direction is achieved by adding attracting goal $g$ in an appropriate location $\mathbf{c}_{g}^{i}$ with $d_{g}^{i}=1$. The example of possible directions for $k_{d}=8$ is presented in Figure 1 .

The above decision making process should be applied only to the node $D_{i}$ which location $c^{i}$ is one of the vertices of convex hull created by $C^{+}$(lies on the estimated boundary). The node that is inside the estimated boundary should move towards one of the edges of approximated cloud's shape. To 


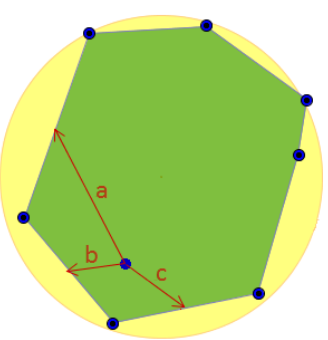

Fig. 2: The target edges chosen by the node inside estimated boundary according to the strategy: (a) — the longest, (b) the closest, (c) - the greatest relation of the length of the edge to the distance to the edge.

improve uniform distribution of nodes on the boundary the target $g$ is located in the middle of the chosen edge. Three strategies for choosing an edge were proposed (Fig. 2):

(a) the longest (to distribute nodes uniformly on the boundary);

(b) the closest (to limit energy consumption);

(c) with the greatest value of ratio length of the edge to distance of the node to the middle of the edge (trade off between energy consumption and uniform distribution).

When the node is on the boundary of the real cloud its greedy decision to increase area of convex hull causes that it escapes from the area covered by gas. Then it is attracted by centroid and returns to the position on the boundary. Such repeated behaviour causes that node pass considerable distance within small area but do not improve quality of cloud detection significantly. Hence, simple stabilization mechanism to limit energy consumption is proposed. If the node senses gas in the given time and was outside the cloud (did not sense gas) in one of the last $k_{s}$ steps then it freezes $\left(\epsilon_{g}^{i}=0^{1}\right)$. The higher value of $k_{s}$ is the greater network stability is expected.

\section{B. Centroid repulsion}

The second, as well decentralized, method is based on idea to repeal nodes that sense gas from the center of cloud to keep track of changing cloud's shape. To repel the node to the boundary of cloud the goal $g$ is localized in $\mathbf{c}_{c}$. The reference distance of that goal $\hat{d}_{g}^{i}$ is slightly greater than the greatest distance between any of points from $C^{+}$and $\mathbf{c}_{c}$ :

$$
\hat{d}_{g}^{i}=\max _{\mathbf{c}^{i} \in C^{+}} d\left(\mathbf{c}^{i}, \mathbf{c}_{c}\right)+\epsilon
$$

where $\epsilon$ is slightly greater than zero and $d\left(\mathbf{c}^{i}, c_{c}\right)$ is Euclidean distance between two points $\mathbf{c}^{i}$ and $\mathbf{c}_{c}$. The result of the repulsion from the centroid is the node movement in direction shown on Fig. 3.

Similarly to greedy approach, when the node is on the boundary it is frequently being pushed out and drag into the cloud. Hence, the aforementioned stabilization mechanism is added also in this case.

\footnotetext{
${ }^{1}$ It is assumed that if $U^{i}=0$ than the node $D_{i}$ freezes.
}

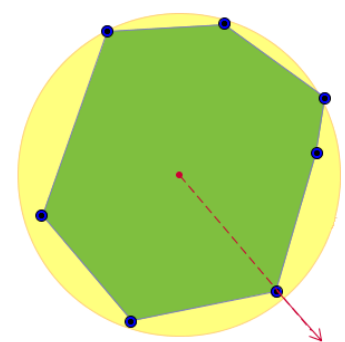

Fig. 3: The force acting on the node in the centroid approach. Red dot is the estimated centroid of the cloud, red arrow represents the force.

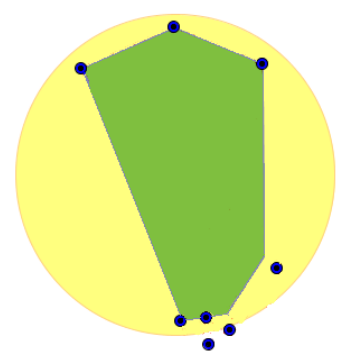

Fig. 4: Distribution of nodes after 40 steps of exemplary simulation if only goal localized in centroid is considered and other nodes' locations are ignored (i.e. $\epsilon_{j}^{i}=0$ ).

If the node location is not influenced by locations of other nodes (i.e. $\epsilon_{j}^{i}=0$ ) then estimated area covered by gas is strictly dependant on initial location of nodes. If the location of nodes is similar, which is a frequent situation in real life scenarios, the nodes are not uniformly distributed on the boundary, thus much of dangerous area is not discovered (Fig. 4). To uniformly distribute nodes on the boundary in decentralized manner each node should repel from each node that senses gas. Hence, $\epsilon_{j}^{i}$ should be greater than zero if node $D_{j}$ senses gas and the expected distance between node $D_{i}$ and $D_{j}\left(d_{i}^{j}\right)$ should be greater than current distance between these nodes $\left(\hat{d}_{j}^{i}\right)$. As long $\hat{d}_{j}^{i}-d_{i}^{j}=\Delta d=\operatorname{const}(\Delta d>0)$ for each node $D_{j}$ it can be observed that according to (1) the closer the nodes are the bigger influence on each other they have. Fig. 5 depicts forces acting on exemplary node from all other nodes and the goal located in centroid, as all are treated as potential sources.

Due to the introduction of stabilization mechanism the question when $\epsilon_{j}^{i}>0$ arises. Four variants exist:

(i) only when repelling from centroid is considered $\left(\epsilon_{g}^{i}>0\right)$;

(ii) only in stabilization phase - when repelling from centroid is deactivated $\left(\epsilon_{g}^{i}=0\right)$;

(iii) always (independently on value of $\epsilon_{g}^{i}$ );

(iv) never.

It can be expected that the more often interaction between nodes is taken into account the better shape of cloud will be estimated. However, the consequence of often interaction 


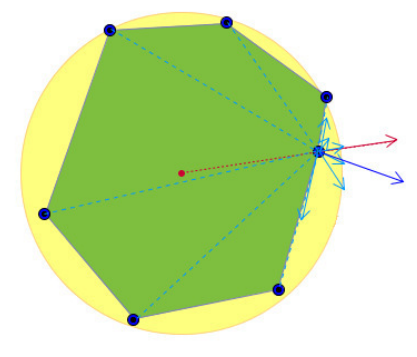

Fig. 5: Forces acting on the node if repelling from other nodes is considered. Red dot is the centroid of estimated cloud area, red arrow is force resulting from repelling from the centroid and blue arrows are forces resulting from repelling from other nodes. Dark blue arrow is resultant force acting on the node.

between nodes will be increased energy consumption due to longer distance traveled by nodes and more communication. All variants, as well as other ideas presented in this section for both greedy and centroid approach, were examined and the results are presented below.

\section{EXPERIMENTS}

The experiments were conducted with MobASim platform [47], which contains both PFM model and simple model of heavy gas dispersion. The aim of the experiments was to verify the quality of the proposed approaches and to adjust their parameters according to the following criteria:

1) percent $C_{c o v}$ of the gas cloud covered by a MANET

$$
C_{\text {cov }}=\frac{\operatorname{cov}_{\mathcal{G}}}{\operatorname{cov}_{G C}} \cdot 100 \%,
$$

where $C_{c o v}$ is the percent of a surface of a gas cloud detected by MANET, $\operatorname{cov}_{G C}$ denotes a surface of the whole gas cloud, $\operatorname{cov}_{\mathcal{G}}$ an area of a polygon with boundary discovered by all sensing devices.

2) total distance traveled by all nodes, which is the major factor of the energy consumption.

Each experiment was composed of nine different (in terms of wind velocity and cloud initial position) simulations in which the MANET was used to detect area covered with gas (Fig. 6). The variety of scenarios allowed for gathering results independent on initial position of network relative to the cloud. In each simulation the same configuration of environment and gas (chlorine) are taken (Table I). In every experiment each of the nodes used the same configuration of the method in the process of network self-organization. The network is composed of $n=10$ nodes.

The result of each experiment are graphs of the above criteria versus time (in each simulation $t=0$ is the moment of detecting the gas by at least one node).

\section{A. Greedy approach}

Firstly, the strategies for choosing target edge by node inside the detected area were examined. The other parameters of this method were set as follows: $k_{d}=8$ and $k_{s}=4$. The results are

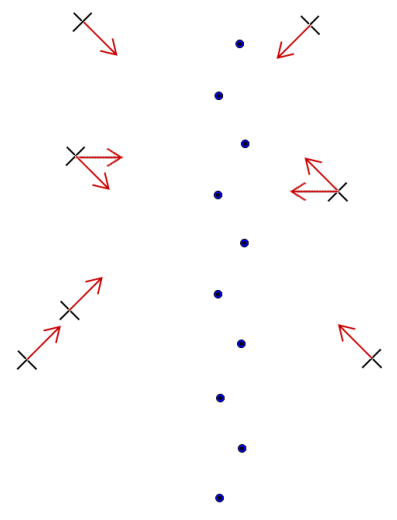

Fig. 6: Initial topology of MANET and configurations of clouds - initial position of cloud is marked with a black cross and the velocity of wind with a red arrow. If one cross is associated with two different arrows then it is two different configurations (with the same initial position of the cloud).

TABLE I: Values of parameters of the chlorine gas cloud simulation model [47].

\begin{tabular}{|c|c|c|}
\hline Symbol & Value & Units \\
\hline $\mathbf{c}_{c}$ & {$[200,200]$} & {$[\mathrm{m}, \mathrm{m}]$} \\
\hline$\left|v_{c}\right|$ & 1 & $\frac{\mathrm{m}}{\mathrm{s}}$ \\
\hline$r$ & 10 & $\mathrm{~m}$ \\
\hline$m_{0}$ & 2000 & $\mathrm{~kg}$ \\
\hline$m_{a}$ & 0 & $\mathrm{~kg}$ \\
\hline$g$ & 9.81 & $\frac{\mathrm{m}}{\mathrm{s}^{2}}$ \\
\hline$\rho_{\text {air }}$ & 1.20 & $\frac{\mathrm{kg}}{\mathrm{m}^{3}}$ \\
\hline$M$ & 71 & $\frac{\mathrm{g}}{\mathrm{mol}}$ \\
\hline$M_{\text {air }}$ & 29 & $\frac{\mathrm{g}}{\mathrm{mgl}}$ \\
\hline$c_{p}$ & 0.48 & $\frac{\mathrm{kJ}}{\mathrm{kg} * K}$ \\
\hline$c_{p}^{\text {air }}$ & 1.01 & $\frac{\mathrm{kJ}}{\mathrm{kg*K}}$ \\
\hline$u_{*}$ & 0.15 & $\frac{\mathrm{m}}{\mathrm{s}}$ \\
\hline$\Delta H_{0}$ & 661 & $\frac{\mathrm{kJ}}{\mathrm{kg}}$ \\
\hline$T_{\text {air }}$ & 293.15 & $\mathrm{~K}$ \\
\hline$\alpha$ & 0.9 & - \\
\hline
\end{tabular}

presented in Figure 7. As expected the quality of gas cloud detection is directly proportional to the distance traveled by nodes. Hence the choice of strategy depends on priorities in concrete application - if the priority is quality of detection than (a) strategy should be chosen and this strategy is chosen for further considerations. Otherwise, if the energy efficiency is critical aspect the (b) strategy is the most efficient choice.

The second parameter to adjust was the number of directions considered by a node localized on the estimated boundary of cloud, $k_{d}$. The length of stabilization phase was set as before to $k_{s}=4$. The obtained results (Fig. 8) for $k_{d} \in\{4,8,16\}$ shows that influence of this parameter on the traveled distance is minimal. However, greater number of considered directions (8 and 16) increases quality of detection. Because of no difference between results for $k_{d}=8$ and $k_{d}=16$, the $k_{d}=8$ is deemed as better as less computation is needed to take single decision.

Finally length of stabilization phase (parameter $k_{s}$ ) was 
(a)

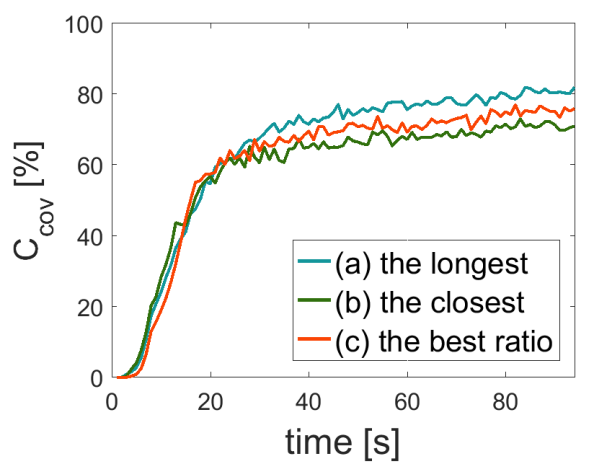

(b)

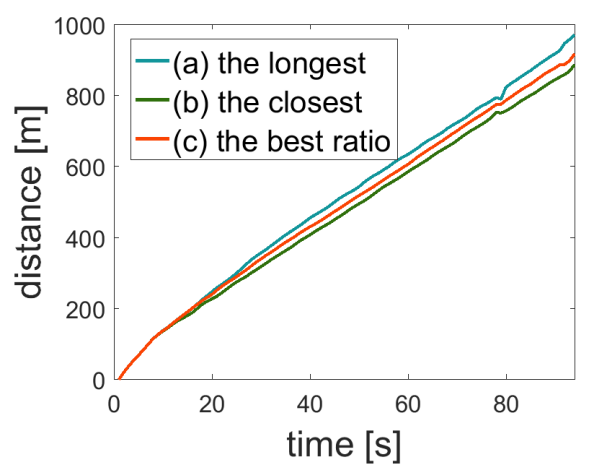

Fig. 7: Cloud detection quality and distance traveled if nodes take decision with greedy approach configured with $k_{d}=8$ and $k_{s}=4$ and different strategies for choosing edge.

considered. As Figure 9 depicts, the stabilization mechanism has great influence on distance traveled - for $k_{s}=4$ the increase of traveled distance is about $45 \%$, whereas decrease in quality of finally detected area covered by cloud is minimal. This value is assumed to be the best in further considerations. According to the results disabling stabilization mechanism can be justified for cases when faster detection of covered area is critical.

The result of deploying MANET composed of nodes taking decision with greedy approach $\left(k_{s}=4, k_{d}=8\right.$ and choosing the middle of the longest edge) at the end of simulation is presented on Figure 10.

\section{B. Centroid repulsion}

Then centroid repulsion approach was examined. In the first experiment the influence of stabilization mechanism and its length (parameter $k_{s}$ ) was tested (Fig. 11), with $\epsilon_{j}^{i}=0$. Significant influence of the stabilization mechanism on the quality of cloud shape detection can be observed only in a very beginning of simulation, when the cloud grows rapidly as expected the longer stabilization period is the smaller area covered by gas is detected. Comparing the distance traveled for different configurations it appears that $k_{s}>4$ does not reduce the energy consumption enough to justify weaker cloud detection. For $k_{s}=4$ the reduce of distance traveled is around $50 \%$ and this value is used in further considerations. (a)

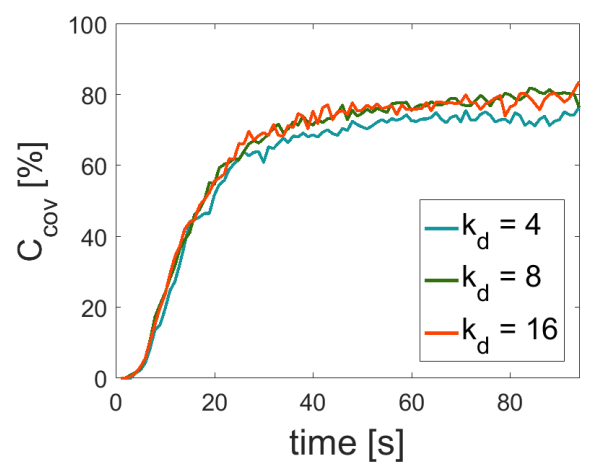

(b)

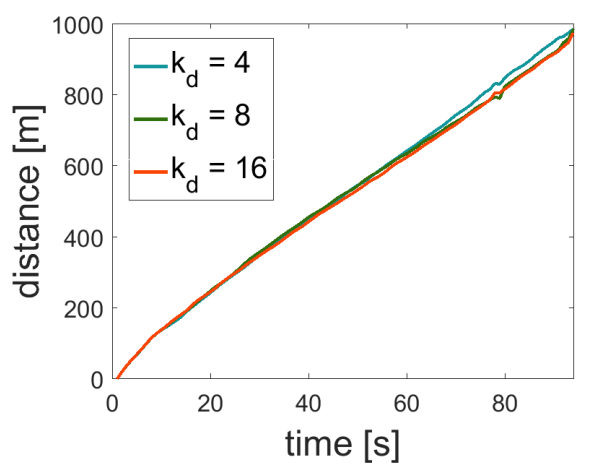

Fig. 8: Cloud detection quality and distance traveled if nodes take decision with greedy approach configured with $k_{s}=4$, choosing the longest edge and different values of $k_{d}$.

The second experiment was conducted to examine the influence of repelling nodes from each other. The results of implementing aforementioned strategies (Fig. 12) indicate significant improvement in cloud detection quality if repelling is turned on $\left(\epsilon_{j}^{i}>0\right)$. However, activating repelling from other nodes in stabilization phase (strategies (ii) and (iii)) causes serious increases of distance traveled by nodes. Hence strategy (i) - repelling from other nodes only when repelling from centroid is active $\left(\epsilon_{g}^{i}>0\right)$ - can be deemed as conciliation between energy and quality aspects. In the future the possibility of changing strategy during the network deployment to increase quality of detection without significant increase of the distance traveled should be examined.

In Figure 13 the topology of network created by nodes that takes decisions according to centroid approach $\left(k_{s}=4\right.$ and repelling from others nodes only when repelling from centroid is active - strategy (i)) is presented.

\section{Comparison}

The detection of the exact boundary of the cloud is impossible due to the limited number of nodes. Hence, it can be assumed that both methods detects the area covered by gas satisfactorily - the nodes are placed uniformly on the boundary, thus detected area is close to the maximum. The greedy approach performs slightly better in the middle phase of the cloud detection (steps 15-60) in terms of detection 
(a)

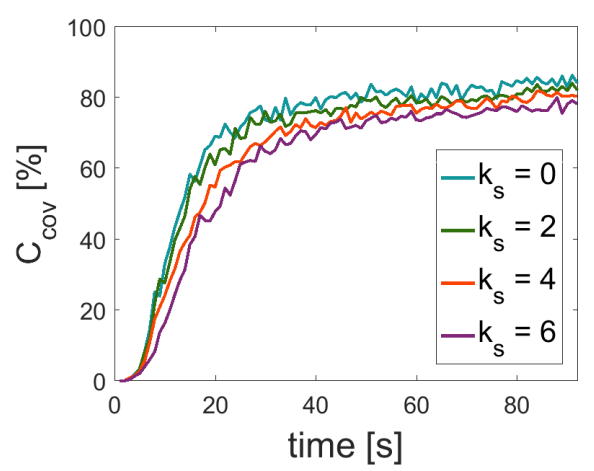

(b)

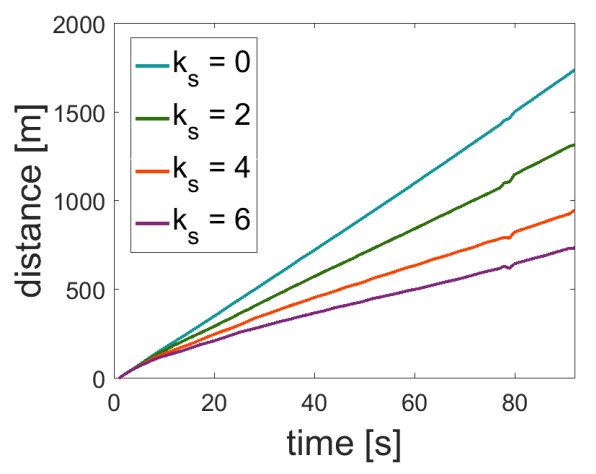

Fig. 9: Cloud detection quality and distance traveled if nodes take decision with greedy approach configured with $k_{d}=8$, choosing the longest edge and different values of $k_{s}$.

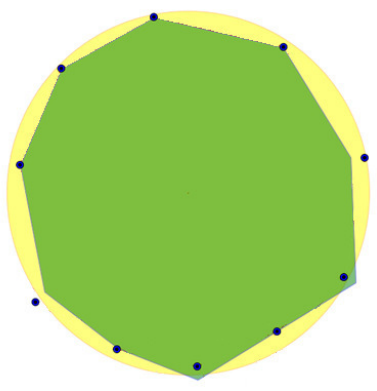

Fig. 10: Topology of MANET in the end of simulation greedy approach with $k_{d}=8$, choosing the longest edge strategy and $k_{s}=4$.

quality (Fig. 14). In respect of energy saving there is no significant difference between both methods.

\section{CONCLUSIONS}

The application of MANET for detecting and tracking cloud of heavy gas was proposed. Two decision-making methods, based on greedy approach and centroid repulsion, were proposed. Both methods proved to be satisfactory in detecting and tracking gas cloud in a simple scenario. Different configurations of methods where tested and compared to adjust parameters values in order to decrease energy consumption and increase quality of cloud's shape detection. (a)

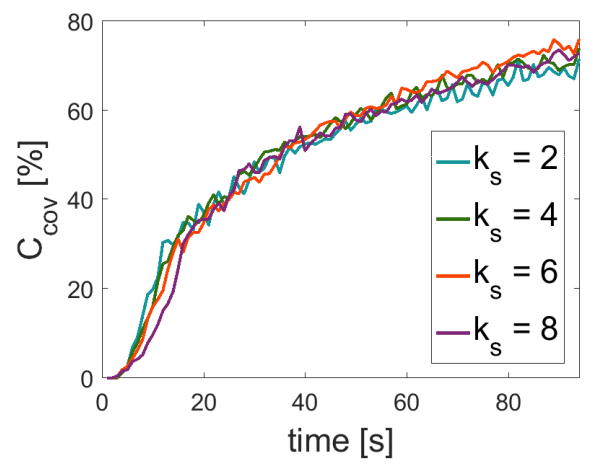

(b)

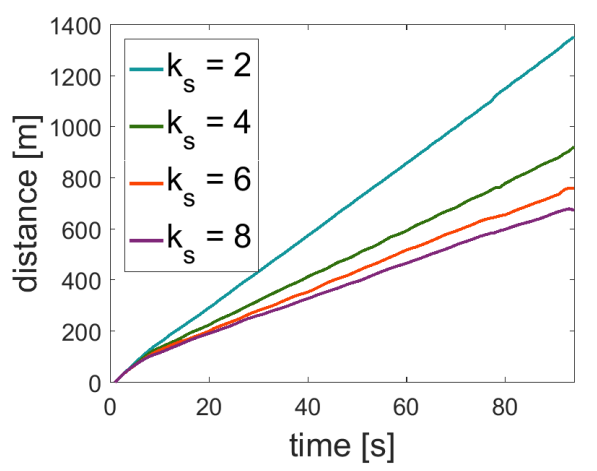

Fig. 11: Cloud detection quality and distance traveled if nodes take decision with centroid approach configured with no repelling from other nodes and different values of $k_{s}$.

We believe that method based on repulsion from centroid should be used as the basis for future research as this method allow straightforward integration with other decision modules (e.g. module to avoid collisions or tracking the boundary), especially if they are based on PFM model as well.

The future research should concentrate on few areas. Firstly, more complex environment should be considered, e.g. with obstacles, slopes and changing wind direction, to examine method in the case of irregular cloud shape. The possibility to dynamically adjust values of discussed parameters during MANET deployment is also worth considering.

Secondly, scenarios without external communication system should be considered. Thus, the method should be extended with connectivity maintenance mechanism which would allow multi-hop communication between each pair of nodes. As presented approaches create ring topology, which can be ineffective and error prune in terms of communication, creating some internal-cloud communication layer or network clusterization should be investigated.

\section{REFERENCES}

[1] F. Scargiali, E. D. Rienzo, M. Ciofalo, F. Grisafi, and A. Brucato, "Heavy gas dispersion modelling over a topographically complex mesoscale: A $\{$ CFD $\}$ based approach," Process Safety and Environmental Protection, vol. 83, no. 3, pp. 242 - 256 , 2005. doi: http://dx.doi.org/10.1205/psep.04073. [Online]. Available: http://www.sciencedirect.com/science/article/pii/S095758200571243X 
(a)

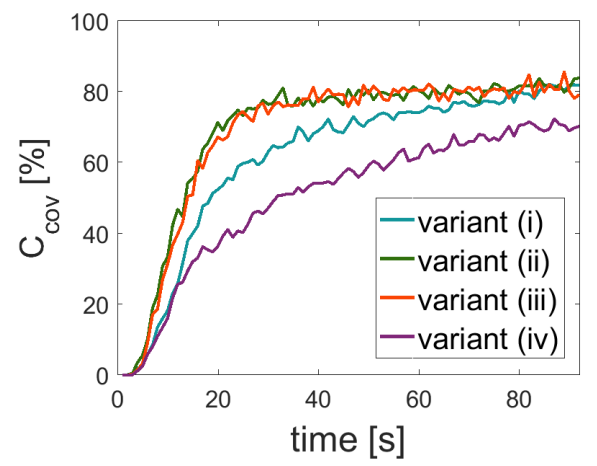

(b)

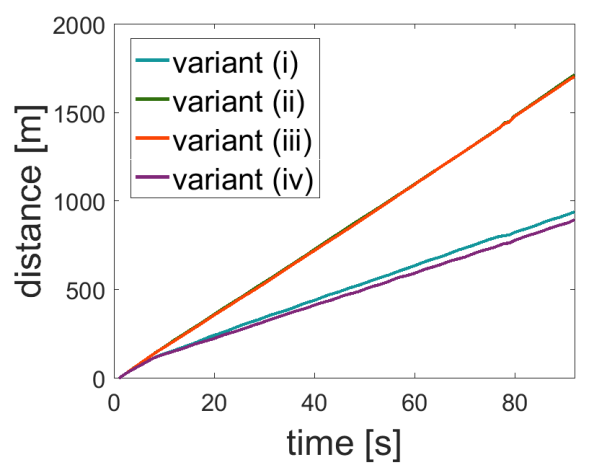

Fig. 12: Cloud detection quality and distance traveled if nodes take decision with centroid approach configured with $k_{s}=4$ and different strategies of repelling from other nodes.

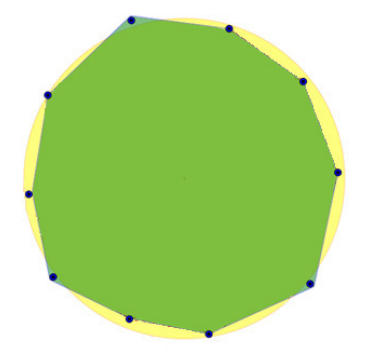

Fig. 13: Topology of MANET in the end of simulation centroid approach with repelling from others nodes only when repelling from centroid is active and $k_{s}=4$.

[2] R. Jones, B. Wills, and K. C., "Chlorine gas: An evolving hazardous material threat and unconventional weapon," Western Journal of Emergency Medicine: Integrating Emergency Care with Population Health vol. 11, no. 2, pp. 151-156, May 2010. doi: 10.1109/TIE.2012.2196010

[3] C. C. Yockey, B. M. Eden, and R. B. Byrd, "The McConnell missile accident. Clinical spectrum of nitrogen dioxide exposure," JAMA, vol. 244, no. 11, pp. 1221-1223, Sep 1980.

[4] N. B. Charan, C. G. Myers, S. Lakshminarayan, and T. M. Spencer, "Pulmonary injuries associated with acute sulfur dioxide inhalation," Am. Rev. Respir. Dis., vol. 119, no. 4, pp. 555-560, Apr 1979.

[5] P. J. Baxter, M. Kapila, and D. Mfonfu, "Lake nyos disaster, cameroon, 1986: the medical effects of large scale emission of carbon dioxide?" BMJ, vol. 298, no. 6685, pp. 1437-1441, 1989. doi: 10.1136/bmj.298.6685.1437

[6] G. J. Fitzgerald, "Chemical warfare and medical response during world war i," American journal of public health, vol. 98, no. 4, p. 611, 2008 doi: 10.2105/AJPH.2007.11930

[7] R. Saladi, E. Smith, and A. Persaud, "Mustard: a potential agent of (a)
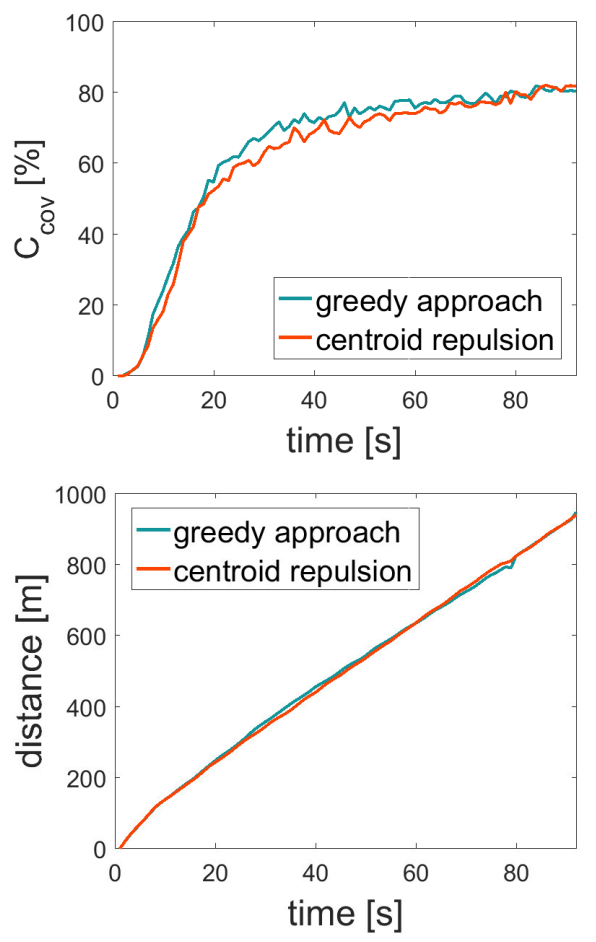

Fig. 14: Comparison of cloud detection quality and distance traveled if nodes take decision with greedy approach $\left(k_{d}=8\right.$, choosing the longest edge and $k_{s}=4$ ) and centroid repulsion approach $\left(k_{s}=4\right.$ and repelling from others nodes only when repelling from centroid is active - variant (i)).

chemical warfare and terrorism," Clinical and experimental dermatology, vol. 31, no. 1, pp. 1-5, 2006.

[8] M. Markiewicz, "Mathematical modeling of heavy gas atmospheric dispersion over complex and obstructed terrain," Archives of Environmental Protection, vol. Vol. 36, no. 1, pp. 81-94, 2010.

[9] Y.-N. Lien, H.-C. Jang, and T.-C. Tsai, "A manet based emergency communication and information system for catastrophic natural disasters," in 29th IEEE International Conference on Distributed Computing Systems Workshops, 2009. ICDCS Workshops '09., June 2009. doi: 10.1109/ICDCSW.2009.72. ISSN 1545-0678 pp. 412-417.

[10] Y.-N. Lien, L.-C. Chi, and C.-C. Huang, "A multi-hop walkie-talkie-like emergency communication system for catastrophic natural disasters," in 39th International Conference on Parallel Processing Workshops (ICPPW), 2010, Sept 2010. doi: 10.1109/ICPPW.2010.77. ISSN 15302016 pp. 527-532.

[11] M. Aloqaily, S. Otoum, and H. Mouftah, "A novel communication system for firefighters using audio/video conferencing/sub-conferencing in standalone manets," in 5th International Conference on Computer Science and Information Technology (CSIT), 2013, March 2013. doi: 10.1109/CSIT.2013.6588764 pp. 89-98.

[12] J. Kim, D. Kim, S. Jung, M. Lee, K. Kim, C. Lee, J. Nah, S. Lee, J. Kim, W. Choi, and S. Yoo, "Implementation and performance evaluation of mobile ad hoc network for emergency telemedicine system in disaster areas," in Engineering in Medicine and Biology Society, 2009. EMBC 2009. Annual International Conference of the IEEE, Sept 2009. doi: 10.1109/IEMBS.2009.5333889. ISSN 1557-170X pp. 1663-1666.

[13] E. Kulla, R. Ozaki, A. Uejima, H. Shimada, K. Katayama, and N. Nishihara, "Real world emergency scenario using manet in indoor environment: Experimental data," in Ninth International Conference on Complex, Intelligent, and Software Intensive Systems (CISIS), 2015, July 2015. doi: 10.1109/CISIS.2015.49 pp. 336-341.

[14] T. Aurisch and J. Tölle, "Relay placement for ad-hoc networks in crisis and emergency scenarios," in Proceedings of the Information Sys- 
tems and Technology Panel Symposium (IST-091), Bucharest, Romania, vol. 11, 2009.

[15] A. Martín-Campillo, R. Martí, S. Robles, and C. Martínez-García, "Mobile agents for critical medical information retrieving from the emergency scene," in 7th International Conference on Practical Applications of Agents and Multi-Agent Systems (PAAMS 2009), ser. Advances in Intelligent and Soft Computing, Y. Demazeau, J. Pavón, J. Corchado, and J. Bajo, Eds. Springer Berlin Heidelberg, 2009, vol. 55, pp. 30-39. ISBN 978-3-642-00486-5. [Online]. Available: http://dx.doi.org/10.1007/978-3-642-00487-2_4

[16] R. Martí, S. Robles, A. Martín-Campillo, and J. Cucurull, "Providing early resource allocation during emergencies: The mobile triage tag," Journal of Network and Computer Applications, vol. 32, no. 6, pp. 1167 - 1182, 2009. doi: http://dx.doi.org/10.1016/j.jnca.2009.05.006. [Online]. Available: http://www.sciencedirect.com/science/article/pii/S1084804509000769

[17] Y. Hayakawa, K. Mori, Y. Ishida, K. Tsudaka, T. Wada, H. Okada, and K. Ohtsuki, "Development of emergency rescue evacuation support system in panic-type disasters," in Consumer Communications and Networking Conference (CCNC), 2012 IEEE, Jan 2012. doi: 10.1109/CCNC.2012.6181047 pp. 52-53.

[18] T. Tsunemine, E. Kadokawa, Y. Ueda, J. Fukumoto, T. Wada, K. Ohtsuki, and H. Okada, "Emergency urgent communications for searching evacuation route in a local disaster," in Consumer Communications and Networking Conference, 2008. CCNC 2008. 5th IEEE, Jan 2008. doi: 10.1109/ccnc08.2007.267 pp. 1196-1200.

[19] T. Nakamura, K. Kogo, J. Fujimura, K. Tsudaka, T. Wada, K. Ohtsuki, and H. Okada, "Development of emergency rescue evacuation support system (eress) in panic-type disasters: Disaster detection by positioning area of terminals," in 42nd International Conference on Parallel Processing (ICPP), 2013, Oct 2013. doi: 10.1109/ICPP.2013.111. ISSN 0190-3918 pp. 931-936.

[20] M. Zhong and C. Cassandras, "Distributed coverage control and data collection with mobile sensor networks," Automatic Control, IEEE Transactions on, vol. 56, no. 10, pp. 2445-2455, Oct 2011. doi: 10.1109/TAC.2011.2163860

[21] M. T. Thai, R. Tiwari, R. Bose, and A. Helal, "On detection and tracking of variant phenomena clouds," ACM Trans. Sen. Netw., vol. 10 no. 2, pp. 34:1-34:33, Jan. 2014. doi: 10.1145/2530525. [Online]. Available: http://doi.acm.org/10.1145/2530525

[22] V. Subramanian, A. Umbarkar, and A. Doboli, "Decentralized detection and tracking of emergent kinetic data for wireless grids of embedded sensors," in Conference on Adaptive Hardware and Systems (AHS), 2012 NASA/ESA. IEEE, 2012. doi: 10.1109/AHS.2012.6268650 pp. 198-204.

[23] Y. Liu and M. Li, "Iso-map: Energy-efficient contour mapping in wireless sensor networks," in 27th International Conference on Distributed Computing Systems, 2007. ICDCS '07., June 2007. doi 10.1109/ICDCS.2007.115. ISSN 1063-6927 pp. 36-36.

[24] J.-H. Kim, K.-B. Kim, C. S. Hussain, M.-W. Cui, and M.-S. Park, "Energy-efficient tracking of continuous objects in wireless sensor networks," in Ubiquitous Intelligence and Computing. Springer, 2008, pp. 323-337.

[25] S. Duttagupta, K. Ramamritham, and P. Ramanathan, "Distributed boundary estimation using sensor networks," in IEEE International Conference on Mobile Adhoc and Sensor Systems (MASS), 2006, Oct 2006. doi: 10.1109/MOBHOC.2006.278571 pp. 316-325.

[26] H. Hong, S. Oh, J. Lee, and S.-H. Kim, "A chaining selective wakeup strategy for a robust continuous object tracking in practical wireless sensor networks," in IEEE 27th International Conference on Advanced Information Networking and Applications (AINA), 2013. IEEE, 2013. doi: 10.1109/AINA.2013.131 pp. 333-339.

[27] K. Matsuo, K. Goto, A. Kanzaki, T. Hara, and S. Nishio, "Overhearingbased efficient boundary detection in dense mobile wireless sensor networks," in IEEE 15th International Conference on Mobile Data Management (MDM), 2014, vol. 1. IEEE, 2014. doi: 10.1109/MDM.2014.34 pp. $225-234$

[28] G. Keung, B. Li, Q. Zhang, and H.-D. Yang, "The target tracking in mobile sensor networks," in Global Telecommunications Conference (GLOBECOM 2011), 2011 IEEE, Dec 2011. doi: 10.1109/GLOCOM.2011.6134188. ISSN 1930-529X pp. 1-5.

[29] S. Srinivasan, S. Dattagupta, P. Kulkarni, and K. Ramamritham, "A survey of sensory data boundary estimation, covering and tracking techniques using collaborating sensors," Pervasive and Mobile Computing, vol. 8, no. 3, pp. 358-375, 2012. doi: 10.1016/j.pmcj.2012.03.003
[30] J. Brink and E. Pebesma, "Plume tracking with a mobile sensor based on incomplete and imprecise information," Transactions in GIS, vol. 18, no. 5, pp. 740-766, 2014. doi: 10.1111/tgis. 12063

[31] T. Sun, H. Pei, Y. Pan, and C. Zhang, "Robust adaptive neural network control for environmental boundary tracking by mobile robots," International Journal of Robust and Nonlinear Control, vol. 23, no. 2, pp. 123-136, 2013. doi: 10.1002/rnc.1816. [Online]. Available: http://dx.doi.org/10.1002/rnc.1816

[32] Z. Jin and A. Bertozzi, "Environmental boundary tracking and estimation using multiple autonomous vehicles," in 46th IEEE Conference on Decision and Control, 2007, Dec 2007. doi: 10.1109/CDC.2007.4434857. ISSN 0191-2216 pp. 4918-4923.

[33] A. Joshi, T. Ashley, Y. R. Huang, and A. L. Bertozzi, "Experimental validation of cooperative environmental boundary tracking with onboard sensors," in American Control Conference, 2009. ACC'09. IEEE, 2009. doi: 10.1109/ACC.2009.5159837 pp. 2630-2635.

[34] J. Brink, "Boundary tracking and estimation of pollutant plumes with a mobile sensor in a low-density static sensor network," Urban Climate, pp. -, 2014. doi: http://dx.doi.org/10.1016/j.uclim.2014.07.002. [Online]. Available: http://www.sciencedirect.com/science/article/pii/ S2212095514000492

[35] S. Susca, F. Bullo, and S. Martínez, "Monitoring environmental boundaries with a robotic sensor network," Control Systems Technology, IEEE Transactions on, vol. 16, no. 2, pp. 288-296, 2008. doi 10.1109/TCST.2007.903395

[36] J. Clark and R. Fierro, "Mobile robotic sensors for perimeter detection and tracking," ISA transactions, vol. 46, no. 1, pp. 3-13, 2007. doi: 10.1016/j.isatra.2006.08.001

[37] D. Marthaler and A. L. Bertozzi, "Collective motion algorithms for determining environmental boundaries," Autonomous Robots, special issue on Swarming, submitted for publication, 2003.

[38] I. Triandaf and I. B. Schwartz, "A collective motion algorithm for tracking time-dependent boundaries," Mathematics and Computers in Simulation, vol. 70, no. 4, pp. 187 - 202, 2005. doi: http://dx.doi.org/10.1016/j.matcom.2005.07.001. [Online]. Available: http://www.sciencedirect.com/science/article/pii/S0378475405001850

[39] S. Srinivasan, "Contour estimation using collaborating mobile sensors," in In DIWANS '06: Proceedings of the 2006 workshop on Dependability issues in wireless ad hoc networks and sensor networks. ACM, 2006. doi: $10.1145 / 1160972.1160986$ pp. 73-82.

[40] M. Kemp, A. L. Bertozzi, and D. Marthaler, "Multi-uuv perimeter surveillance," in Proceedings of, 2004. doi: 10.1109/AUV.2004.1431200 pp. $102-107$.

[41] S. Subchan, B. A. White, A. Tsourdos, M. Shanmugavel, and R. Zbikowski, "Dubins path planning of multiple uavs for tracking contaminant cloud," in Proceedings of the 17th World Conference on the International Federation of Automatic Control, Seoul, Korea, 2008. doi: 10.3182/20080706-5-KR-1001.00964 pp. 6-11.

[42] B. White, A. Tsourdos, I. Ashokaraj, S. Subchan, R. Żbikowski et al., "Contaminant cloud boundary monitoring using network of uav sensors," Sensors Journal, IEEE, vol. 8, no. 10, pp. 1681-1692, 2008. doi: 10.1109/JSEN.2008.2004298

[43] A. Sinha, A. Tsourdos, and B. White, "Multi $\{\mathrm{UAV}\}$ coordination for tracking the dispersion of a contaminant cloud in an urban region," European Journal of Control, vol. 15, no. 3-4, pp. 441 - 448, 2009. doi: http://dx.doi.org/10.3166/ejc.15.441-448. [Online]. Available: http://www.sciencedirect.com/science/article/pii/S0947358009709999

[44] D. W. Casbeer, R. W. Beard, T. W. McLain, S.-M. Li, and R. K. Mehra, "Forest fire monitoring with multiple small uavs," in American Control Conference, 2005. Proceedings of the 2005. IEEE, 2005. doi: 10.1109/ACC.2005.1470520 pp. 3530-3535.

[45] S. Srinivasan, K. Ramamritham, and P. Kulkarni, "Ace in the hole: Adaptive contour estimation using collaborating mobile sensors," in International Conference on Information Processing in Sensor Networks, 2008. IPSN'08. IEEE, 2008. doi: 10.1109/IPSN.2008.38 pp. 147-158.

[46] E. Niewiadomska-Szynkiewicz, A. Sikora, and J. Kołodziej, "Modeling mobility in cooperative ad hoc networks," Mobile Networks and Applications, vol. 18, no. 5, pp. 610-621, 2013. doi: 10.1007/s11036013-0450-2

[47] A. Sikora, E. Niewiadomska-Szynkiewicz, and M. Krzyszton, "Simulation of mobile wireless ad hoc networks for emergency situation awareness," in Federated Conference on Computer Science and Information Systems (FedCSIS), 2015. IEEE, 2015. doi: 10.15439/2015F52 pp. 1087-1095. 